\title{
Chemotherapiefreie, kombinierte Erhaltung bei metastasiertem Darmkrebs machbar
}

\begin{abstract}
Die gleichzeitige Hemmung des vaskulären endothelialen Wachstumsfaktors (VEGF) und des epidermalen Wachstumsfaktorrezeptors (EGFR) hat sich beim metastasierten kolorektalen Karzinom (mCRC) in der Erstlinie nicht bewährt. Doch wie sieht es mit dieser Kombination als Erhaltungstherapie aus?
\end{abstract}

$\mathrm{n}$ einer randomisierten Phase-III-Studie wurde eine Erhaltungstherapie mit dem EGFR-Inhibitor Erlotinib und dem VEGF-Antikörper Bevacizumab bei Patienten mit nicht resektablem mCRC geprüft, die nach einer Bevacizumab-basierten Induktionstherapie nicht progredient waren. Sie erhielten weiter eine niedrige Bevacizumab-Dosis (7,5 mg/kg alle 3 Wochen) entweder allein oder in Kombination mit Erlotinib $(150 \mathrm{mg} / \mathrm{d}$ ) bis zum Progress. Von 700 initial eingeschlossenen Patienten waren nach der Induktion 452 progressionsfrei und konnten randomisiert werden: 228 in die Bevacizumab-Gruppe, 224 in die Gruppe mit Bevacizumab plus Erlotinib.
In der primären Analyse nach 231 Ereignissen hatte das progressionsfreie Überleben (PFS, primärer Endpunkt) bei 5,1 bzw. 6,0 Monaten gelegen (stratifizierte Hazard Ratio [HR] 0,79, $95 \%$-Konfidenzintervall [95\%-KI] 0,601,$06 ; \mathrm{p}=0,11$; unstratifizierte HR 0,76, $95 \%$-KI 0,59-0,99; $p=0,043)$. In der finalen Analyse nach median 51,0 Monaten in der Monotherapie- und 48,3 Monaten in der Kombinationsgruppe lag das PFS in dem jeweiligen Arm bei median 5,4 und 4,9 Monaten. Die stratifizierte HR betrug hier $0,81(\mathrm{p}=0,059)$, die unstratifizierte HR 0,78 ( $\mathrm{p}=0,019)$.

Beim Gesamtüberleben war in der finalen Analyse die Kombinationsgrup- pe signifikant überlegen mit median 24,9 vs. 22,1 Monaten seit Randomisierung (stratifizierte HR 0,79, 95\%-KI 0,63-0,99; $\mathrm{p}=0,036$; unstratifizierte HR $0,79,95 \%$-KI 0,64-0,98; $\mathrm{p}=0,035)$. Allerdings war die Kombination aus Bevacizumab plus Erlotinib mit deutlich mehr Hautausschlägen des Grads 3/4 assoziiert als die Monotherapie (21 vs. $0 \%$ ). Auch Diarrhö (10 vs. 1\%) und Asthenie ( 5 vs. $<1 \%$ ) waren häufiger.

Fazit: Die geprüfte Kombination könnte eine neue chemotherapiefreie Option für eine Erhaltungstherapie beim nicht operablen mCRC nach Bevacizumab-haltiger Induktionstherapie in der ersten Linie sein. Es bleibt die Abwägung von Zeitgewinn und einem Mehr an Toxizität, zumal das PFS nur um im Median 0,5 Monate verlängert wurde.

Friederike Klein

Tournigand C et al. Bevacizumab with or without erlotinib as maintenance therapy in patients with metastatic colorectal cancer (GERCOR DREAM; OPTIMOX3): a randomised, open-label phase 3 trial. Lancet Oncol. 2015;16(15):1493-505.

\section{Neoadjuvante Therapie bei Rektumkarzinom keine Frage des Alters}

Fehlende Studiendaten mit älteren Patienten sollten nicht zum Vorenthalten der Therapie nur wegen des Alters führen. Darauf weist eine Datenbankauswertung von Patienten mit lokal fortgeschrittenem Rektumkarzinom (LARC) erneut hin.

D Forscher prüften den Einfluss des Alters auf die Ergebnisse bei neoadjuvanter Strahlenchemotherapie von Patienten mit LARC, die zwischen 2005 und 2012 in einem von 5 kanadischen Zentren in kurativer Absicht operiert worden waren.

Für die Analyse von krankheitsfreiem Überleben (DFS), krebsspezifischem Überleben (CSS) und Gesamtüberleben (OS) wurde zwischen unter 70 -jährigen und älteren Patienten unterschieden. Es flossen aber auch andere wichtige prognostische Faktoren mit in die Auswertung ein, z. B. Geschlecht, Allgemeinzu- stand, Stadium, Abstand des Tumors vom Anus etc.

Von 1.172 auswertbaren Patienten waren 295 (25\%) 70 Jahre und älter. Sie erhielten seltener eine adjuvante Chemotherapie (ACT; 60 vs. $79 \%$; $<0,0001$ ), insbesondere eine Oxaliplatin-basierte ACT (12 vs. $31 \%$; p < 0,0001) und schlossen seltener eine neoadjuvante Chemotherapie ab (76 vs. $86 \%, p<0,001)$. Außerdem waren sie häufiger bei Beginn der neoadjuvanten Strahlenchemotherapie anämisch (42 vs. $30 \%, \mathrm{p}=0,0004$ ).

In der multivariaten Analyse war aber ein Alter von 70 Jahren und mehr assoziiert mit einem vergleichbaren DFS wie bei jüngeren Patienten (Hazard Ratio [HR] 0,93, 95\%-Konfidenzintervall [95\%-KI] 0,68-1,26; $\mathrm{p}=0,63]$. Das galt auch für das CCS (HR 0,81, $95 \%$-KI $0,46-1,41 ; \mathrm{p}=0,45)$ und das OS (HR $1,28,95 \%$-KI $0,88-1,86$; $\mathrm{p}=0,20)$. Wurde das Alter als eine kontinuierliche Variable zugrundegelegt, war ein zuneh- mendes Alter nicht prädiktiv für das DFS (HR 1,00, 95\%-KI 0,99-1,02; $\mathrm{p}=0,49$ ) oder das CSS (HR 1,002, $95 \%$-KI 0,98-1,02; $\mathrm{p}=0,88)$, wohl aber für ein geringeres OS (HR 1,02, $95 \%$-KI $1,00-1,03 ; \mathrm{p}=0,04)$.

Fazit: Patienten im Alter von 70 Jahren und mehr profitieren von der neoadjuvanten Strahlenchemotherapie mit nachfolgender chirurgischer Behandlung ähnlich wie jüngere Patienten. Therapieentscheidungen hinsichtlich neoadjuvanter Strahlenchemotherapie und Chirurgie sollten daher nicht lediglich auf Basis des Alters getroffen werden. Allerdings sind Studien zur Risikostratifizierung in der sehr heterogenen Gruppe älterer Patienten nötig.

Friederike Klein

Jiang DM et al. Clinical outcomes of elderly patients receiving neoadjuvant chemoradiation for locally advanced rectal cancer. Ann Oncol. 2015;26(10):2102-6. 